\section{MS23-P8 TEM characterization of hydroxyapatite nanocrystals grown on the bacterial cellulose fibrils}

Natalya A. Arkharova ${ }^{1}$, Alexander V. Severin ${ }^{2}$, Albert K. Khripunov $^{3}$, Andrey S. Orekhov ${ }^{1}$, Vera V. Klechkovskaya ${ }^{1}$

1. Institute of Crystallography, RAS, 119333, Leninskii pr. 59, Moscow, Russia

2. Department of chemistry, Moscow State University, 119991, 1-3 Leninskiye Gory, Moscow, Russia

3. Institute of Macromolecular Compounds, RAS, 199004, Bol'shoi pr. 31, St. Petersburg, Russia

email: natalya.arkharova@gmail.com

Hydroxyapatite (HAP) nanocrystals were synthesized by the biomimetic approach (simulation of the biosynthesis in natural bone): synthesis of HAP nanocrystals occurred in the presence of bacterial cellulose fibrils. Due to its properties (biocompatibility, elasticity, high wet strength, conformability, high modulus value) and crystalline structure bacterial cellulose (BC) is promising collagen substitute material. Composites were prepared with the different BC/HAP mass ratios by gradual increasing of $\mathrm{BC}$ portion up to maximum possible: $\quad$ BC/HAP_1 - 0,04/1 g, BC/HAP_2 -0,24/1 g, BC/HAP_3 - 0,79/1g, BC/HAP_4 $1 / 1 \mathrm{~g}$. Microstructure of the samples was investigated with transmission electron microscopy (TEM) and electron diffraction. High-resolution TEM (HRTEM) and HRTEM simulation by the Bloch wave method were used to determine the thickness of the HAP nanocrystals. In all prepared samples HAP nanoparticles are textured along [0001] direction to the $\mathrm{BC}$ fibril direction similarly to alignment of mineral nanocrystals on collagen fibrils in natural bone (Fig.1.). It was determined that the more mass proportion of the $\mathrm{BC}$ is in the solution than there are less big HAP nanoparticles in the sample and their sizes are close to sizes of mineral crystals in bone.

This work was supported by grant RFBR № 14-02-31258.

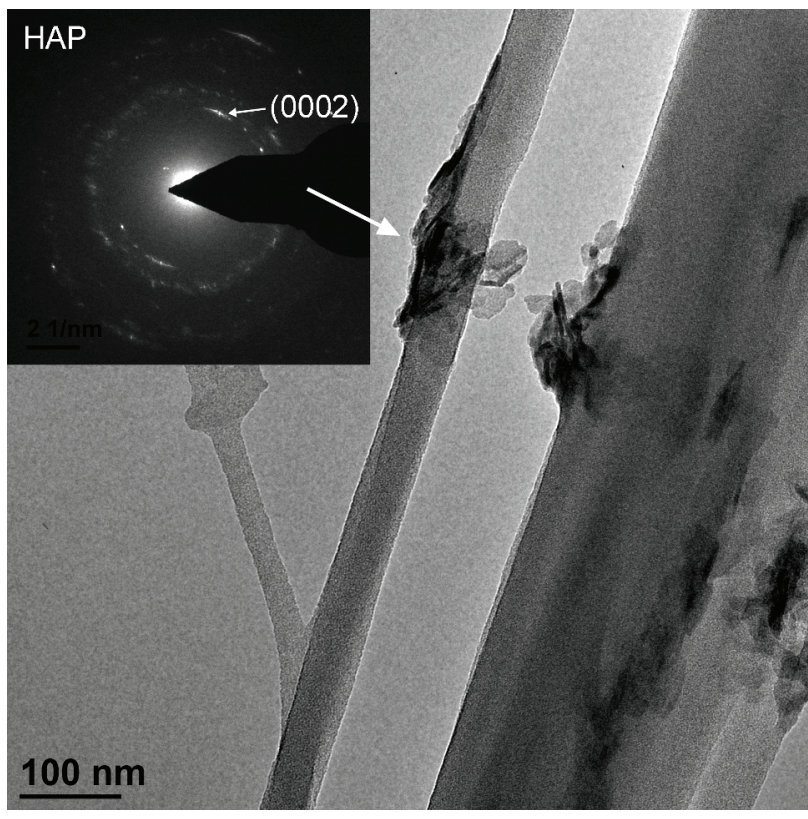

Figure 1. TEM image of HAP agglomerate grown on the bacterial cellulose fibril and its diffraction pattern.

Keywords: bacterial cellulose, hydroxyapatite nanoparticles, electron microscopy, electron diffraction 